\title{
The Affects of Lightning Strikes and High Current on Polymer Composites
}

L. M. Gammon, * and B. S. Hayes**

*Boeing Materials Technology, P.O. 3707 MC 73-44, Seattle, Washington 98124

**University of Washington, Department of Chemical Engineering, Seattle, WA, 98195

This paper will describe the macro and micro effects of lightning strikes and resulting high current, high energy arcing on aerospace polymeric composite materials. Changes to the composite microstructure will be shown from laboratory generated lightening strikes and the result of high current affects as it travels though the composite and the transition zone caused by high energy arcing.

Modern aircraft are constructed of a wide range of structural materials, which include a variety of metals and composites. Because of the diversity in these materials, it is important to understand and document all the microstructural changes that can occur when there is an arcing event. When an arc occurs involving annealed steel, an untempered martensite marbled in a matrix of ferrite due to the instantaneous heating and rapid quenching by the surrounding material is seen.

Composite conductivity, however, is not as homogenous as in metals. Although there are entrance and exit zones (as the case for metals) and carbon fibers are very conductive, they are surrounded by an insulator (the polymer resin). Therefore the current travels differently through the material. Unique to carbon fibers there is an interply arcing as the current jumps from ply to ply.

The macro affects of a lightning strike on composites are usually apparent and can be documented with macrophotography but the micro affects can only be observed with a clean cross-section of the area. The zone in and around the strike is very fragile due to the vaporized matrix as shown in Figure 1 and 2, respectively. Care must be taken in cross-sectioning and mounting in order to preserve much of the critical information. Mounting and sectioning lightning strike composite specimens are best accomplished with a two-stage mount. It is advisable on the primary mount to add a dye to the mounting epoxy, such as rhodamine-B laser dye. The best method for creating an artifact free specimen is to first vacuum impregnate the strike area with the epoxy followed by a pressure cure. This encapsulated area will allow a section through the center of the strike and thus hold the fragile material in place, minimizing artifacts. The specimen can be remounted to provide adequate handling of the microscopic sample.

The microstructural analysis can be observed with the full range of optical micro-tools: bright field, polarized light, dark field, and epi-fluorescence. In polymer composites, however, you may observe hot spots or interply and intraply arcing (Figure 3), unlike metals with their multiphase eutectic melted zone. The microstructure of polymer composites that have been exposed to high energy arcing is quite different and can vary widely depending on the polymer. The strike affects in thermoset polymers usually have a central hot spot zone where the fibers are degraded and surrounded by a zone where the polymer is vaporized. The bulk structure of the surrounding polymer can also change depending on the polymer matrix (single phase or 
toughened multiphase). The use of a laser dyed back filled epoxy is very important so that the vaporized zone is preserved and apparent with the fluorescence, leaving no doubt which are the composite polymer and the mounting polymer (see Figure 3).
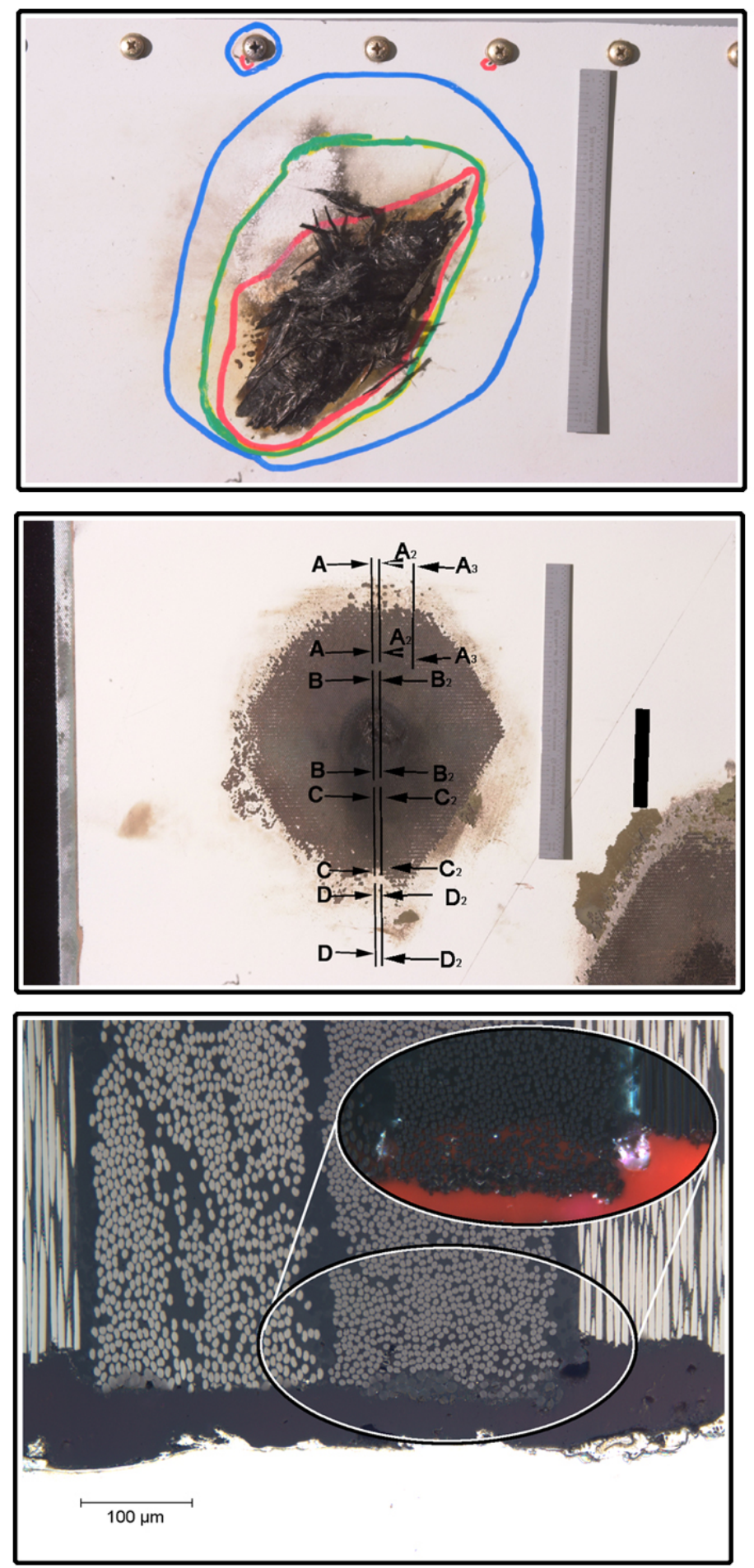

Figure 1: A lab induced lightning strike on a carbon fiber composite.
Figure 2: A section map of a lab induced lightning strike on a honeycomb carbon fiber composite structure that contained an expanded wire aluminum mat.
Figure 3. A carbon fiber composite showing a hot spot due to intraply arcing. It has been backfilled with rhodamine B laser dyed epoxy. This shows the voided zone adjacent and around the center of the hot spot. Polarized light illumination. 